\title{
Role of tipranavir in treatment of patients with multidrug-resistant HIV
}

This article was published in the following Dove Press journal:

Therapeutics and Clinical Risk Management

8 September 2010

Number of times this article has been viewed

\author{
Joshua D Courter ${ }^{1,2}$ \\ Colleen J Teevan' \\ Michael $\mathrm{H} \mathrm{Li}^{1}$ \\ Jennifer E Girotto ${ }^{1,2}$ \\ Juan C Salazar ${ }^{1,2,3}$
}

'University of Connecticut School of Pharmacy, Storrs, ${ }^{2}$ Connecticut Children's Medical Center, Hartford, ${ }^{3}$ University of Connecticut School of Medicine, Hartford, Connecticut, USA
Abstract: The worldwide emergence of multidrug-resistant human immunodeficiency virus (HIV)-1 strains has the driven the development of new antiretroviral (ARV) agents. Over the past 5 years, HIV-entry and integrase inhibitor ARVs, as well as improved non-nucleoside reverse transcriptase inhibitors (NRTIs) and protease inhibitors (PIs), have become available for treatment. It is important to assess how these new ARVs might be most judiciously used, paying close attention to viral susceptibility patterns, pharmacodynamic parameters, and the likelihood that patients will adhere to their therapy. Herein we review published material in Medline, EMBASE, and ISI for each antiretroviral agent/classes currently approved and summarize the available data on their efficacy, safety, and pharmacologic parameters. We focus on the role of tipranavir, a recently approved nonpeptidic PI, for treating HIV-infected children, adolescents, and adults with a history of multidrug-resistant HIV.

Keywords: tipranavir, HIV, multidrug resistance

\section{Introduction}

One in 155 adults and adolescents are infected with human immunodeficiency virus (HIV) globally, with an astonishing rate of one in 21 in Sub-Saharan Africa. ${ }^{1}$ In the US alone, more than half a million people were living with HIV in 2007, and 44,000 new cases were reported in the same year. ${ }^{2}$ Although safe and effective antiretroviral (ARV) treatment is now available, the prevalence of genotypic resistance to protease inhibitors (PIs) and non-nucleoside reverse transcriptase inhibitors (NNRTIs) has steadily increased. ${ }^{3}$ In treatment-naïve adolescents, genotypic resistance has been reported to be as high as $15 \% .{ }^{4}$ Given this scenario it is now more important than ever to understand how newer ARV agents may provide therapeutic alternatives for treatment-naïve and treatment-experienced HIV-infected patients.

The appearance of HIV-resistant strains for the most part is due to the development of point mutations in the HIV genome. Because HIV reverse transcriptase lacks the proofreading abilities of human DNA polymerase, 0.2 to 1 new point mutations may appear during each viral replication cycle. ${ }^{5}$ This translates into approximately 10,000 new mutations per day. Fortunately for the host, most of these mutations are lethal to the virus and thus do not result in added ARV resistance. ${ }^{5}$ Resistance only occurs when the mutation induces conformational changes which allow the virus to overcome the action of the inhibiting agent, while maintaining the activity of the enzyme. When HIV does develop a beneficial point mutation, the corresponding genetic sequence is amplified by selective pressures of ARV therapy. These genetic changes in the viral genome and their effects are also the basis for available resistance testing.
Correspondence: Juan C Salazar Connecticut Children's Medical Center Division of Pediatric Infectious Diseases 2L, 282 Washington St, Hartford, CT 06106, USA

Tel +I 8605459490

$\mathrm{Fax}+\mathrm{I} 860545$ 937I

Email jsalaza@ccmckids.org 


\section{Resistance testing}

Resistance testing helps to direct therapy by avoiding the use of ineffective or inappropriate ARV therapy. For this reason the current adult and adolescent HIV-1 guidelines recommend resistance testing for all new patients entering into care, HIV-positive women who are pregnant or planning to become pregnant, and when considering changes to a patient's ARV regimen due to suboptimal viral load reduction or virologic failure. ${ }^{6}$ The pediatric HIV guidelines also recommend resistance testing prior to starting therapy in treatment-naïve children, for treatment failure, and when considering the start of a CCR 5 antagonist. ${ }^{7}$

HIV-resistance patterns can be assessed by using either a genotypic assay or by determining its phenotypic susceptibility to ARVs. Genotypic assays screen viral genes for mutations which confer resistance to specific ARVs or entire classes of agents. This is done by either genomic mapping or the use of a DNA probe. ${ }^{6}$ The resulting list of mutations is interpreted using a regularly updated database containing mutations known to confer ARV resistance. ${ }^{8}$ Phenotypic testing involves the use of different concentrations of specific ARVs against the virus in vivo. ${ }^{6}$ Results of this test indicate the concentration at which $50 \%$ of the HIV viral replication is inhibited in culture $\left(\mathrm{IC}_{50}\right) \cdot{ }^{6} \mathrm{~A}$ combination of phenotypic and genotypic tests, known as virtual phenotyping, has also been used. ${ }^{9}$ This method involves the use of a database, which matches a genotypic pattern to a known phenotypic profile. ${ }^{9}$

Rates of virologic success, defined as achieving a viral load of $<200$ copies/mL of blood, where compared between patients randomized to ARV therapy directed by genotyping, phenotyping, or standard of care (NARVAL trial). ${ }^{10,11}$ Patients allocated to therapy chosen by genotypic analysis (44/183) trended toward increased virologic success when compared with those whose therapy was chosen by phenotyping $(36 / 183 ; P=0.068)$ or standard of care $(36 / 152$; $P=0.052) .{ }^{10}$ Using multivariate analysis, patients in the genotypic regimen arm were found to have a higher likelihood of achieving virologic success than patients receiving standard of care (odds ratio [OR] 2.13 (95\% confidence interval $[\mathrm{CI}] 1.20-3.79 ; P=0.01)] .{ }^{11}$ In another study, ARV treatment was chosen according to virtual phenotype and then compared with treatment chosen based on genotype in 327 adult patients. ${ }^{12}$ At 48 weeks, no differences were found between patients achieving undetectable viral load in the virtual phenotype group and the genotype group ( $42 \%$ and $46 \%$, respectively; $P=0.553$ ). ${ }^{12}$ These discrepant results indicate that there are benefits and limitations to each type of resistance testing. ${ }^{13}$ Nevertheless, the adult and adolescent guidelines show preference for genotypic testing for treatment-naïve and pregnant patients. ${ }^{6}$

\section{HIV life cycle and mechanism of drug action}

In order to understand how ARVs function it is important to understand the HIV life cycle. The first step of viral entry is for HIV to locate a T lymphocyte via the CD4 receptor site. ${ }^{14}$ After the $\mathrm{T}$ lymphocyte is bound, the virus must also bind to either a CCR5 (site of action of maraviroc) or CXCR4 coreceptor. ${ }^{15}$ The viral membrane then proceeds to fuse with the host cell membrane, via GP 41 (site of action of efuvirtide), enabling its RNA and proteins to enter the T lymphocyte. Inside the cell, viral reverse transcriptase produces HIV DNA using the viral RNA as a template (site of action of NRTIs and NNRTIs). Integrase enzyme then incorporates the HIV DNA into the human cell's genome through several steps (site of action of integrase inhibitors). Viral DNA is then transcribed by human RNA polymerase, producing viral RNA. This RNA is translated into polyprotein precursors, which are then cleaved by protease into functional proteins (site of action of PIs).

\section{Newer ARV treatment options for HIV-positive adults and children}

There are several new ARV classes, as well as new agents from the older classes. In this study we performed an extensive literature search using Medline, EMBASE, and ISI, and collected data regarding efficacy, safety, and pharmacologic parameters of the relevant agents.

\section{CCR5 antagonists}

Maraviroc is the only currently approved CCR5 antagonist in clinical use. This agent acts through allosteric inhibition of the CCR5 receptor, preventing its interaction with gp120 and thus avoiding subsequent cellular entry by HIV. Data from two clinical trials, Maraviroc versus Optimized Therapy in Viremic Antiretroviral Treatment-Experienced Patients (MOTIVATE-1 [US and Canada; $\mathrm{n}=585$ ] and MOTIVATE-2 [US, Australia, and Europe; $n=464]$ ), have been published. ${ }^{16}$ The study inclusion criteria were identical in both trials. Patients at least 16 years of age were enrolled into the studies if they were infected with a CCR5-tropic HIV strain and were receiving at least one NRTI, or one NNRTI, or a fusion inhibitor, two PIs, and had documented resistance to at least three of the aforementioned classes. ${ }^{16}$ When administered with an optimized background therapy (OBT), more patients in the combined once-daily 
or twice-daily maraviroc arms achieved an undetectable viral load ( $<50$ copies $/ \mathrm{mL})$, than patients receiving placebo $(43 \%$, $46 \%$, and $17 \%$, respectively, $P<0.001) .{ }^{16}$

CCR5 antagonists only work against M-tropic strains of HIV, which are typically found in the early stages of infection. ${ }^{17}$ This is due to their exclusive use of CCR5 coreceptors, rather than CXCR4, which is found in later stages of HIV. ${ }^{17}$ Therefore, maraviroc should only be used in patients with CCR5-tropic strains, and in not those which are CXCR4-tropic, dual-tropic, or mixed-tropic.

The most common adverse events reported for maraviroc were diarrhea, nausea, headache, fever, fatigue, and upper respiratory tract infection. ${ }^{16}$ Laboratory abnormalities were similar to those in the placebo arm. ${ }^{16}$ Maraviroc does carry a black box warning for hepatotoxicity preceded by an allergic reaction, as this had been reported in a healthy volunteer study.

Maraviroc has been approved by the US Food and Drug Administration (FDA) and European Medicines Agency (EMA) for use in patients with multidrug-resistant CCR5tropic strains of HIV-1. ${ }^{18,19}$ A study evaluating safety, pharmacokinetics, and efficacy is currently enrolling children 2-18 years of age infected with CCR5-tropic HIV-1. ${ }^{20}$ Maravirocbased regimens are not currently recommended for children younger than 16 years of age or for treatment-naïve patients. ${ }^{6}$ It is, however, recommended for use in treatment-experienced adults and adolescents infected with CCR5-tropic HIV-1 experiencing failure on their current regimen. ${ }^{6}$

Maraviroc is currently available as $150 \mathrm{mg}$ and $300 \mathrm{mg}$ tablets. A pediatric liquid formulation is currently under development. ${ }^{18-20}$ The monthly cost of maraviroc therapy is approximately $\$ 1000$ USD. Because maraviroc is only efficacious in patients with CCR5-tropic HIV-1 strains, the adult and adolescent HIV-1 guidelines recommend a coreceptor tropism assay be performed prior to treatment initiation or upon failure while on maraviroc. ${ }^{6}$ The cost of the CCR5 tropism assay (about $\$ 1960$ ) could limit the ability to screen patients for the use of maraviroc. ${ }^{21}$

\section{Fusion inhibitors}

One single fusion inhibitor, enfuvirtide, is approved for clinical use. Enfuvirtide acts by binding to a region of the HIV-1 gp41 envelope protein, resulting in its inhibition, and thereby inhibits the steps which lead to fusion of the viral envelope and the cell membrane. ${ }^{22}$ Due to its unique mechanism of activity, enfuvirtide maintains activity against isolates resistant to other ARV classes.

The efficacy of enfuvirtide was studied in two internationally conducted clinical trials of identical design, ie, the
T-20 versus Optimized Regimen Only studies (TORO-1 [US, Canada, Mexico, and Brazil; $\mathrm{n}=501$ ] and TORO-2 [Australia and Europe; $\mathrm{n}=512]) .{ }^{23,24}$ Patients 16 years of age and older who had previously documented ARV resistance, or who had received a minimum of 6 months of at least one NRTI and NNRTI, and at least two PIs, were eligible for participation. Patients were randomized to receive enfuvirtide plus an OBT or OBT alone. By 24 and 48 weeks, more patients in the enfuvirtide group achieved an undetectable viral load $(<50$ copies $/ \mathrm{mL})$ than in the placebo group $(15.9 \%$ versus $6.3 \%$ and $18.3 \%$ versus $7.8 \%$, respectively). ${ }^{25}$

The most prevalent mutations associated with enfuvirtide resistance result from an alteration of the active site of gp41. ${ }^{26}$ Resistance against enfuvirtide is possible not only through mutations in gp41, but also in gp $120 .{ }^{27}$ The most commonly reported adverse events experienced by the enfuvirtide group were injection site reaction, nausea, vomiting, fatigue, headache, insomnia, and peripheral neuropathy. ${ }^{28}$ Pneumonia and lymphadenopathy occurred at significantly higher rates in the enfuvirtide group. Hypersensitivity reactions can also occur. ${ }^{28}$

Enfuvirtide has been approved for use in treatmentexperienced patients 6 years of age and older infected with HIV-1 in both Europe and in the US. ${ }^{18,19}$ Enfuvirtide is not recommended for use in treatment-naïve children, but should be considered as an additional ARV in patients who have failed NRTI, NNRTI, and PI regimens. ${ }^{7}$ Because enfuvirtide is a synthetic peptide, and therefore cannot be administered orally, it is currently available as a $90 \mathrm{mg} / \mathrm{mL}$ subcutaneous injection. The monthly cost of enfuvirtide is \$2500 USD.

\section{Nucleoside reverse transcriptase inhibitors}

This class consists of DNA base analogs, which are reversible competitive inhibitors of reverse transcriptase, effectively preventing reverse transcription of viral RNA into DNA. ${ }^{29}$ There are currently 11 FDA-approved ARVs in this class. ${ }^{18}$ Dual NRTI therapy is recommended as part of therapy for treatmentnaïve patients. ${ }^{6}$ The primary mechanism of resistance to NRTIs involves conformational changes at the active site. ${ }^{30}$ In a recent study from the US and Europe, 6.1\% and 3.8\%, respectively, had NRTI resistance mutations. ${ }^{31}$ The overall incidence of NRTI resistance has decreased now that patients use this class of ARV in combination with NNRTIs and/or PIs.

\section{Non-nucleoside reverse transcriptase inhibitors}

NNRTIs act noncompetitively to inhibit the formation of DNA from viral RNA. ${ }^{32}$ Resistance to NNRTIs occurs when 
mutations confer structural changes around the allosteric binding site of reverse transcriptase. ${ }^{33}$ In a study of 413 US and 500 Western European HIV-positive ARV-naïve patients, $7.3 \%$ and $3.2 \%$, respectively, had NNRTI resistance mutations. ${ }^{31}$

There are currently four NNRTIs approved by the FDA, including delavirdine, efavirenz, nevirapine, and etravirine. As a class, NNRTIs have a lower genetic barrier to resistance than PIs, but are a line of therapy which can spare the use of PIs until later in life, ${ }^{6,7}$ and have much fewer drug interactions, as well as gastrointestinal and lipid-related adverse reactions. ${ }^{6}$ Of the available agents, efavirenz is recommended as first-line, with the exception of use in patients who are pregnant or likely to become pregnant, for whom nevirapine is preferred. ${ }^{6}$ For children under 3 years of age, nevirapine is preferred, because it is available in a liquid formulation. ${ }^{7}$ Efavirenz is the preferred NNRTI for use in HIV-infected children over the age of 3 years. Although not obtainable in a liquid formulation, it is available in capsules sized for pediatric dosing. ${ }^{7}$ As a class, the most common side effects of NNRTIs are rash, central nervous system effects, and lipoatrophy. ${ }^{6}$

Etravirine is the newest NNRTI. It is active against both wild-type and NNRTI-resistant HIV. Resistance is less likely to develop against etravirine because it is a flexible molecule and can bind reverse transcriptase in multiple conformations. ${ }^{34}$ Decreased response to etravirine was associated with the presence of three or more NNRTI mutations (Val90Ile, Ala98Gly, Leu100Ile, Lys101Glu, Lys101Pro, Val106Ile, Val179Asp, Val179Phe, Tyr181Cys, Tyr181Ile, Tyr181Val, Gly190Ala, and Gly190Ser) concurrently. ${ }^{35}$

HIV-positive patients with documented resistance to NNRTIs, with three or more primary PI mutations and a history of having received 3 or more months of different NRTIs were enrolled into a Phase II etravirine clinical trial. The viral load had to be $>1000$ copies $/ \mathrm{mL}$. Patients were randomly assigned to receive either $400 \mathrm{mg}$ or $800 \mathrm{mg}$ of etravirine twice a day, or to serve as part of the control group. All patients on etravirine received a background regimen of at least two different ARVs (NRTI, lopinavir-ritonavir, or enfuvirtide). Patients in the control group received three or more similar ARVs. After 24 weeks, viral loads decreased by 1.04, 1.18, and $0.19 \log _{10}$ copies $/ \mathrm{mL}$ in the $400 \mathrm{mg}, 800 \mathrm{mg}$, and control groups, respectively. The decrease in viral load was greater in the patients receiving $800 \mathrm{mg}$ of etravirine and those who were not using either enfuvirtide or lopinavir-ritonavir. The percentage of patients who had viral loads $<400$ copies $/ \mathrm{mL}$ at 24 weeks was $30 \%$ in subjects receiving $400 \mathrm{mg}$ of etravirine and $38 \%$ in those receiving $800 \mathrm{mg}$. By comparison, only $7.5 \%$ of subjects in the control group achieved a standard similar viral load. ${ }^{34}$

By 48 weeks, the viral load had decreased from baseline by $0.88,1.01$, and $0.14 \log _{10}$ copies $/ \mathrm{mL}$ in the etravirine 400mg twice daily, etravirine $800 \mathrm{mg}$ twice daily, and control groups, respectively. Approximately $20 \%$ of patients receiving either dose achieved viral loads $<50$ copies $/ \mathrm{mL}$ whereas none of the patients in the control group had viral loads $<50$ copies $/ \mathrm{mL}^{36}$

The DUET-1 trial, a multicenter international trial, enrolled 612 patients on stable ARV therapy for at least 8 weeks prior to screening. To be included in the study, patients had to have a viral load $>5000$ copies $/ \mathrm{mL}$, three or more primary PI mutations, and at least one mutation associated with NNRTI resistance. Patients were randomized to receive etravirine $200 \mathrm{mg}$ or placebo. All patients received a suitable NRTI, ritonavir-boosted darunavir, and also had the option of receiving enfuvirtide as part of their backbone therapy. Primarily as a result of virologic failure, $14 \%$ of patients in the etravirine group and $18 \%$ of patients in the placebo group discontinued participation in the study prior to 24 weeks. After 24 weeks, $56 \%$ of patients in the etravirine group and $39 \%$ in the placebo group had a viral load $<50$ copies $/ \mathrm{mL}$. Of note, $74 \%$ of subjects in the etravirine arm and $51 \%$ of patients in the placebo group achieved a viral load $<400$ copies $/ \mathrm{mL}^{35}$

The DUET-2 trial enrolled 591 patients in Australia, Belgium, Canada, France, Germany, Italy, Netherlands, Poland, Portugal, Spain, the UK, and the US. Patients were randomized to the same treatment or placebo arms as in the DUET-1 trial. Virologic failure led to early discontinuation in $17 \%$ of patients on etravirine and $25 \%$ of patients on placebo. After 24 weeks, $62 \%$ of patients on etravirine and $44 \%$ of those on placebo had achieved a viral load $<50$ copies/ $\mathrm{mL}$. Additionally, $75 \%$ of patients on etravirine achieved a viral load $<400$ copies $/ \mathrm{mL}$ versus $54 \%$ of patients in the placebo group. ${ }^{37}$

Etravirine is currently approved for use in combination with other ARV agents for the treatment of adults who have tried other treatments and have evidence of HIV-1 strains resistant to NNRTIs and other ARV agents. ${ }^{18}$ The monthly cost of etravirine is \$800 USD.

The most common adverse events were diarrhea (22.0\%), injection site reaction (related to enfuvirtide, $20.1 \%$ ), fever (20.1\%), rash (20.1\%), fatigue (15.7\%), headache (15.7\%), and nausea $(15.1 \%)$. Rashes appeared early in therapy, usually during the first 2-4 weeks, and resolved in about 1-2 weeks with continued dosing. Rashes were mild to 
moderate in severity and were not dose-related..$^{36}$ Psychiatric adverse events, including mood swings, nightmares, abnormal dreams, anxiety, and depression were reported in $10.7 \%$ versus $2.5 \%$ of patients on etravirine versus placebo, respectively. These did not lead to any patients discontinuing treatment. Headache and insomnia were the most common adverse events related to the nervous system. ${ }^{34}$

\section{Integrase inhibitors}

Currently, there is only one approved integrase inhibitor, raltegravir, which specifically inhibits strand transfer. ${ }^{38}$ This step is inhibited through allosteric modification of the integrase enzyme, preventing its binding to host DNA. ${ }^{39}$ Inhibition of the other steps of integration is currently in the preclinical research phase.

The efficacy of raltegravir was studied in two Merck Blocking Integrase in Treatment Experienced Patients with a Novel Compound against HIV, (BENCHMRK-1 [Europe, Asia, Australia, and Peru; $\mathrm{n}=344$ ] and BENCHMRK-2 [North and South America; $n=344$ ]) trials, which both used the same study design. ${ }^{40}$ They enrolled patients at least 16 years old with HIV-1, a viral load $>1000$ copies $/ \mathrm{mL}$, and a documented resistance to at least one drug from each of the three major classes (NRTI, NNRTI, and PI). Each patient was randomized to an optimized background regimen or optimized background regimen plus raltegravir. After 16 weeks of therapy, significantly more patients receiving the raltegravir-containing regimen achieved an undetectable viral load (less than 50 copies $/ \mathrm{mL}$ ) than in the placebo group in the BENCHMRK-1 trial (61.8\% versus $34.7 \%$, respectively, $P<0.001) .{ }^{40}$ Similar results were seen in the BENCHMRK-2 trial. ${ }^{40}$ At 48 weeks, a combined analysis showed a significantly higher number of patients with undetectable viral load $(<50$ copies $/ \mathrm{mL})$ in the raltegravir group than in the placebo group (62.1\% versus $32.9 \%$, respectively). ${ }^{40}$

Although the mechanism of resistance is not well understood, it is known that there is no cross-resistance with other ARV classes. Within the class, cross-resistance with as yet unavailable integrase inhibitors appears to be substantial. Additionally, as supported by a Phase III trial, clinical failures were generally seen in patients who had developed a point mutation at one of three amino acid residues. ${ }^{41}$ Specifically, in one trial that enrolled patients older than 16 years showed that $64 \%$ of the 462 raltegravir group versus $34 \%$ of the 228 treatment-experienced patients were counted as treatment failures. ${ }^{40}$

Raltegravir was generally well tolerated in the BENCHMRK trials, with diarrhea, nausea, headache, and fatigue being the most common adverse events. ${ }^{40}$ Elevated total cholesterol and triglyceride levels were the most common laboratory abnormalities reported. It is important to note that elevated creatine kinase levels were observed in the clinical trials, and that cases of myopathy and rhabdomyolysis have been reported since that time. ${ }^{40}$

Raltegravir has FDA and EMA approval for use in HIVinfected adult patients. ${ }^{18,19}$ Raltegravir has also been recently recommended as a preferred regimen for treatment-naïve patients in the current adult and adolescent guidelines, and is considered a fully active ARV agent in patients naïve to integrase inhibitors. ${ }^{6}$ Currently, a Phase I/II trial assessing the efficacy, safety, and pharmacokinetics of raltegravir is enrolling HIV-infected children 4 weeks to 18 years of age. ${ }^{42}$ Raltegravir is presently available in $400 \mathrm{mg}$ tablets, with a pediatric formulation under investigation. ${ }^{18,42}$ The monthly cost of raltegravir is $\$ 972.00$.

\section{Protease inhibitors}

There are currently 10 approved PIs, which are based on an analog of a peptide bond which the protease normally cleaves. ${ }^{43}$ This, in turn, prevents gag and gag-pol polyproteins from being synthesized into structural proteins and viral enzymes. PI-based regimens are known to confer an additional benefit in decreasing the development of genotypic mutations. ${ }^{44-46}$ By decreasing mutation rates, the likelihood of developing new resistance is also decreased.

Resistance to PIs generally involves a mutation affecting the structure of the active site of protease. ${ }^{47}$ In a recent study in ARV-naïve HIV-infected patients from the US and Western Europe, $3.6 \%$ and $0.8 \%$ of screened subjects, respectively, had PI resistance mutations. ${ }^{31}$

Ten PIs have been approved by the FDA, including saquinavir, indinavir, ritonavir, nelfinavir, amprenavir, lopinavir, fosamprenavir, atazanavir, darunavir, and tipranavir. ${ }^{6}$ Of these, ritonavir-boosted atazanvir, darunavir, fosamprenavir, and lopinavir regimens are preferred, with alternative regimens of ritonavir-boosted saquinavir or unboosted fosamprenavir or atazanavir. ${ }^{6}$ Only ritonavir-boosted lopinavir is recommended as a preferred PI for children. ${ }^{7}$ Alternatively, ritonavir-boosted atazanavir or fosamprenavir, as well as unboosted nelfinavir, are recommended. ${ }^{7}$ Of these agents, lopinavir coformulated with ritonavir is available as a pediatric oral solution and as tablets, and is approved for children 2 weeks of age and older. ${ }^{7}$ Fosamprenavir and nelfinavir are also available as pediatric suspensions, and are approved for use in children 2 years of age and older. ${ }^{7}$ Atazanavir is available in a pediatric capsule formulation, and is approved for use in children 6 years of age and older. ${ }^{7}$ 
The PIs are generally known to produce adverse effects related to gastrointestinal intolerance, dyslipidemia, and drug metabolism. ${ }^{6}$ Of the PIs, atazanavir is known to have the fewest effects on lipids. ${ }^{6}$

\section{Darunavir}

Of the PIs, both darunavir and tipranavir are able to maintain antiviral activity against many PI-resistant HIV-1 strains. Resistance to darunavir has been associated with 11 mutations (V11I, V32I, L33F, I47V, I50V, I54L, I54M, G73S, L76V, I84V, and L89V). ${ }^{48}$ One study demonstrated that over $20 \%$ of treatment failures were associated with the V32I and I54L mutations. ${ }^{49}$ Darunavir exhibits high-affinity binding properties, in addition to strong chain interactions and potential hydrogen bonding with both the HIV-1 protease, as well as mutant proteases..$^{50,51}$ This allows for greater activity against resistant strains as compared with other PIs. Furthermore, darunavir-resistant strains need to undergo multiple simultaneous mutations to overcome darunavir binding affinity. ${ }^{50}$

A darunavir-ritonavir combination is FDA-approved for the treatment of HIV infection in children 6 years and older weighing at least $20 \mathrm{~kg} \cdot{ }^{52} \mathrm{In}$ adult and adolescent patients, darunavir-ritonavir is recommended as a preferred PI therapy to start in ARV-naïve patients. ${ }^{6}$ In pediatric patients, darunavir is recommended as a second-line treatment option after failure of initial therapy due to the high pill burden required in children weighing less than $40 \mathrm{~kg} .{ }^{7}$ However, it is also suggested that darunavir may be an option for ARV-experienced pediatric patients. ${ }^{7}$ Currently, darunavir is available as $75 \mathrm{mg}, 150 \mathrm{mg}, 300 \mathrm{mg}, 400 \mathrm{mg}$, and $600 \mathrm{mg} .{ }^{52}$ The monthly cost of darunavir for a patient receiving $600 \mathrm{mg}$ twice daily is $\$ 990$.

The most commonly reported adverse events with darunavir include diarrhea (32\%), nausea (18\%), nasopharyngitis $(12 \%)$, headache $(11 \%)$, and upper respiratory tract infection $(10 \%) .{ }^{53}$

\section{Tipranavir}

Like darunavir, tipranavir is a nonpeptidic PI, with an increased flexibility at the binding portion of the molecule. ${ }^{54}$ This increased flexibility results in a greater ability to overcome conformational changes in the protease enzyme due to mutations. Nonpeptidic PIs also act to prevent dimerization of the protease, preventing its activity through multiple pathways. ${ }^{55}$ These advantages have been demonstrated in its maintenance of a relatively low $\mathrm{IC}_{90}$ when tested against HIV-1 strains which had been made highly resistant to ritonavir. ${ }^{56}$ Interestingly, tipranavir, like other PIs, has been shown to modulate Pneumocystis carinii growth. ${ }^{57}$

Several predictors of response have been evaluated for tipranavir. One of the earliest predictors studied was the number of baseline PI mutations. There are seven mutations which are major determinants of tipranavir resistance, ie, L33F, I47V, Q58E, T74P, V82L, V82T, and I84V. ${ }^{8}$ There are also 14 mutations which play a minor role in conferring tipranavir resistance, ie, L10V, I13V, K20M, K20R, E35G, M36I, K43T, M46L, I54A, I54M, I54V, H69K, N83D, and L90M. ${ }^{8}$ It has been found that patients with viruses with two or fewer universal PI-associated mutations are significantly more likely to achieve virologic response. ${ }^{58,59} \mathrm{~A}$ derivation of counting mutations is the tipranavir mutation score, whereby a genotyping is performed, and the mutations conferring reduced in vitro or in vivo tipranavir susceptibility or response are counted. ${ }^{60}$ This measure was found to be highly variable, and had poor correlation with virologic response. ${ }^{60}$ Patients achieving a trough concentration of at least $15 \mu \mathrm{M}$ achieved a $-1.1 \log _{10}$ viral load reduction. ${ }^{61}$ In a small retrospective analysis of 29 adult patients, tipranavir trough levels were found to be significantly higher in patients achieving a virologic response $(P=0.03) .{ }^{62}$ This, however, was not significantly associated with virologic response when analyzed using logistic regression $(P=0.17) .{ }^{63}$ The unbound tipranavir $\mathrm{IC}_{90}$ and $\mathrm{IC}_{50}$ are $0.1 \mu \mathrm{M}$ and $0.03-0.07 \mu \mathrm{M}$, respectively. ${ }^{54,64}$

Less traditional predictors of response have also been assessed. The genotypic sensitivity score, sometimes referred to as an optimized background score considers the number of drugs in the optimized background regimen which have been shown to be active through genotypic testing. ${ }^{65-67}$ For adult patients with a genotypic sensitivity score of $\geq 2,64.2 \%$ achieved a virologic response, compared with a $20 \%$ virologic response rate for those with a score $<2 .{ }^{66}$ This trend was not observed in pediatric patients, possibly due to the small number of patients enrolled, and very few having a genotypic sensitivity score of $\geq 2 .{ }^{67} \mathrm{An}$ inhibitory quotient is calculated by dividing the tipranavir trough by the tipranavir $\mathrm{IC}_{50}{ }^{68-70}$ A general breakpoint might be thought of as $>50-60$, because two analyses found that most patients (81\%) above this level achieved at least a $-1 \log _{10}$ viral load reduction..$^{68,69}$ Another analysis found that an inhibitory quotient of $\geq 76$ had a response rate of $64 \%$, whereas an inhibitory quotient $<76$ had a response rate of $29 \%{ }^{70}$

The genotypic inhibitory quotient (gIQ) is calculated by dividing the tipranavir serum trough concentration by the number of tipranavir resistance conferring mutations genotyped from the patient's HIV strain. ${ }^{62,63,66,67,70}$ The measure 
of gIQ had the highest correlation with virologic success in both children and adults. When correlating gIQ, tipranavir trough, tipranavir mutation score, use of enfuvirtide, and genotypic sensitivity score with virologic response in adult patients using logistic regression, gIQ was found to be the most significant predictor of virologic response $(P=0.03) .{ }^{63}$ At 48 weeks, a significantly greater reduction in viral load was observed in adult patients who had a gIQ $>14,500 \mathrm{ng} /$ $\mathrm{mL} /$ mutation $(83.3 \%$ versus $38.4 \%) .{ }^{66}$ At 48 weeks, pediatric patients with a gIQ above the first quartile (0.56-7.19) were more likely to achieve a viral load of $<400$ copies/mL $(8 \%$ versus $52-68 \%) .{ }^{67} \mathrm{~A}$ more recent assessment of gIQ found that patients with more than eight tipranavir resistance mutations were unlikely to achieve a virologic response, regardless of the tipranavir trough level. ${ }^{62}$

The pharmacokinetics of a tipranavir-ritonavir combination have been studied in both adult and pediatric patients. Like many other PIs, tipranavir is metabolized via the cytochrome (CYP) p450 3A4 isozyme. ${ }^{71}$ Tipranavir should always be coadministered with ritonavir because of its known inhibition of CYP $3 \mathrm{~A} 4$ metabolism, resulting in a boosting effect. This is important because tipranavir alone strongly induces its own metabolism, producing trough concentrations less than $5 \%$ of those produced by the combination. ${ }^{72}$ The understanding of the overall effects of the tipranavir-ritonavir combination on CYP p450 has been recently expanded to include an initial and steady-state phase. ${ }^{73}$ Upon initiating tipranavir-ritonavir, the ritonavir component results in strong inhibition of hepatic and, possibly, intestinal CYP 3A4 and 5 , along with p-glycoprotein. ${ }^{73}$ This eventually balances with the inductive effects of tipranavir to a moderate inhibition of hepatic and strong inhibition of intestinal CYP 3A4 and 5, with little effect on p-glycoprotein. ${ }^{73}$

Tipranavir is highly protein-bound, with $>99.9 \%$ of the drug bound to albumin or $\alpha$-1-acid glycoproteins. ${ }^{56}$ In order to assure adequate absorption, the capsule formulation of tipranavir should be administered with a high-fat meal. ${ }^{74}$ The oral solution has been found to be unaffected by this dietary consideration. ${ }^{75}$

The primary adult efficacy data comes from two Randomized Evaluation of Strategic Intervention in multiresistant patients with Tipranavir (RESIST-1 [US and Australia; $\mathrm{n}=620$ ] and RESIST-2 [Europe and Latin America; $\mathrm{n}=539$ ]) trials, both of which had the same study design. ${ }^{76,77}$ Patients included were HIV-1 infected adults with a baseline viral load of at least 1000 copies/mL, at least one primary PI mutation corresponding to baseline PI therapy, with no more than two resistance-associated PI mutations. All patients were assessed by clinicians prior to randomization using genotypic testing to determine an appropriate PI, NRTI, and NNRTI regimen. They were then randomized to receive an unblinded regimen of either tipranavir boosted with ritonavir or their previously selected comparator PI. The primary efficacy endpoint measured was the proportion of patients achieving at least a $-1 \log _{10}$ viral load decrease. A higher proportion of patients receiving tipranavir achieved the primary endpoint in both the RESIST-1 (41.5\% versus $22.3 \% ; P<0.0001)$ and RESIST- 2 trials (41\% versus $14.9 \% ; P<0.0001$ ) at 24 weeks.

The efficacy of tipranavir has been studied in pediatric patients from the US, Europe, and Latin America. ${ }^{78}$ This trial enrolled children 2-18 years of age who had a baseline viral load of more than 1500 copies/mL. Unlike the adult trial, treatment-naïve patients were also included and no resistance profile requirements were placed as criteria for enrollment. Children in this study were stratified by age into three categories, ie, 2-5, 6-11, and 12-18 years of age. The primary outcome for this study was safety and therefore it was not designed with sufficient power to measure efficacy outcomes. Nonetheless, viral loads as well as CD4 cell counts, were performed as secondary endpoints. As in the adult trial, patients were assigned an optimized background regimen prior to randomization. Patients in this noncomparator study received an unblinded regimen consisting of an optimized background regimen, including at least two other non-PIs as well as either low- $\left(290 / 115 \mathrm{mg} / \mathrm{m}^{2}\right)$ or high-dose $\left(375 / 150 \mathrm{mg} / \mathrm{m}^{2}\right)$ tipranavir-ritonavir, with an upper dosing limit equal to the adult dose of $500 / 200 \mathrm{mg}$.

At 48 weeks, $42.6 \%$ of all patients achieved a viral load of $<400$ copies/mL. In the high-dose group, $45.6 \%$ achieved viral loads $<400$ copies $/ \mathrm{mL}$ compared with only $39.7 \%$ in the lowdose group. A mean viral load decrease of $1.24 \log _{10}$ copies $/ \mathrm{mL}$ and $0.8 \log _{10}$ copies $/ \mathrm{mL}$ was seen in the high- and low-dose groups, respectively. A trend toward better efficacy was seen in the high-dose group with respect to viral load reduction, although it was not statistically significant. As for immunologic response to therapy, a median increase in $\mathrm{CD}^{+} \mathrm{T}$ cell percentages was seen in both the high- (5\% increase) and low-dose group ( $3 \%$ increase), although these values were not statistically different $(P=0.11)$. The immunologic response to therapy was greatest in the $2-5$ year age group (10\% and $6 \%$ for the low- and high-dose groups, respectively), likely due to the less resistant HIV strains of the younger children. Over the 48-week period, four patients developed AIDS-defining illnesses; all four had been receiving the low-dose regimen. 
The investigators concluded that there was a potential benefit of the high-dose regimen over the low-dose regimen.

A total of 78 study patients were continued on or switched to the high-dose regimen after 48 weeks. An abstract has been published to summarize the long-term safety and efficacy data gained from these patients over 100 weeks of high-dose therapy. ${ }^{79}$ Overall, $38 \%$ of patients maintained a viral load of $<400$ copies $/ \mathrm{mL}$ and $34 \%$ maintained an undetectable viral load of $<50$ copies $/ \mathrm{mL}$. The $2-5$-year-old age group experienced the greatest virologic response, with $56 \%$ achieving a viral load of $<400$ copies $/ \mathrm{mL}$ and $48 \%$ achieving an undetectable viral load of $<50$ copies $/ \mathrm{mL}$.

Tipranavir carries a black box warning for both fatal and nonfatal intracranial hemorrhage, because these have been seen in the adult population, ${ }^{18}$ although these have not been observed in the pediatric or adolescent populations. ${ }^{78}$ Black box warnings also indicate a risk for clinical hepatitis and hepatic decompensation in patients with chronic hepatitis $\mathrm{B}$ or $\mathrm{C}$ coinfection. ${ }^{18}$ This subset of patients has been excluded from pediatric and adolescent tipranavir studies, and should apply to these populations as well. Recently, a study analyzing electrocardiograms demonstrated only clinically insignificant changes in corrected QT interval, with a maximum observation of 8.3 milliseconds for healthy patients receiving a supratherapeutic dose of tipranavir $750 \mathrm{mg} .{ }^{18}$

For the pediatric and adolescent populations, safety endpoints have been reported based on the Division of AIDS standardized toxicity grading table. ${ }^{80}$ The 48 -week safety outcomes were not out of the ordinary, with $53.9 \%$ of patients experiencing a drug-related adverse event, $25.2 \%$ of patients experiencing a serious adverse event, and $8.7 \%$ patients discontinuing use of the study drug due to an adverse event. ${ }^{78}$ The most commonly reported adverse events were nausea, vomiting, diarrhea, and headache. An increased risk of bleeding was also described in the pediatric and adolescent study. ${ }^{78}$ When considering laboratory abnormalities, elevations in gamma-glutamyl transpeptidase and alanine transaminase led to discontinuation of tipranavir in four patients. ${ }^{78}$

A subanalysis from the RESIST trials assessed health-related quality of life in patients receiving tipranavircontaining regimens versus those receiving a comparator protease. ${ }^{81}$ It was found that changes in health-related quality of life from baseline were similar between groups.

Currently, tipranavir in combination with ritonavir is FDA-approved for use in patients 2 years of age and older, who are treatment-experienced and infected with an HIV-1 strain clinically resistant to more than one PI. ${ }^{18}$ The European Medicines Agency has given a positive opinion for tipranavir use in highly pretreated adolescents ( $>12$ years old) with evidence of resistance to multiple PIs, and also in highly pretreated children aged $2-12$ years. The tipranavir-ritonavir combination is not currently recommended as initial therapy according to the adult and adolescent guidelines. ${ }^{6}$

\section{Conclusions}

The tipranavir-ritonavir combination provides another promising line of therapy for patients infected with viruses exhibiting PI resistance. Tipranavir has been proven to be relatively safe in both the adult and pediatric populations, giving a valuable alternative for these patients. The PI class effect in decreasing the formation of new mutations makes tipranavir agent more desirable for HIV strains already highly resistant to other ARV therapies.

\section{Disclosure}

JCS has served as a speaker for the Merck vaccine division, GSK, and Pfizer. He has also served as a consultant to Boehringer Ingelheim and was the principal investigator in the primary tipranavir pediatric clinical trial.

\section{References}

1. World Health Organization. World Health Statistics 2009: Cause of specific mortality and morbidity. Available at: http://www.who.int/whosis/ whostat/EN_WHS09_Table2.pdf. Accessed on Dec 6, 2009.

2. Department of Health and Human Services, Centers for Disease Control and Prevention. Cases of HIV infection and AIDS in the United States and Dependent Areas, 2007. HIV/AIDS surveillance report. 2007; 19:1-63.

3. Wheeler W, Mahle K, Bodnar U, et al. Antiretroviral drug resistance mutations and subtypes in drug-naïve persons newly diagnosed with HIV-1 infection, United States, Mar 2003-Oct 2006. 14th Conference on Retroviruses and Opportunistic Infections (CROI 2007). 2007 Feb 25-28; Los Angeles, CA.

4. Viani R, Peralta A, Aldrovandi G, et al. Prevalence of primary HIV drug resistance among recently infected adolescents; a multicenter adolescent trials network study: ATN029. 13th Conference on Retroviruses and Opportunistic Infections (CROI 2007). 2006 Feb 5-8; Denver, CO.

5. Hu DJ, Dondero TJ, Rayfield MA, et al. The emerging genetic diversity of HIV-the importance of global surveillance for diagnostics, research, and prevention. JAMA. 1996;2759(3):210-216.

6. Panel on Antiretroviral Guidelines for Adults and Adolescents. Guidelines for the use of antiretroviral agents in HIV-1-infected adults and adolescents. Department of Health and Human Services. 2009 Dec 1;p. 1-161. Available at: http://www.aidsinfo. nih.gov/ContentFiles/AdultandAdolescentGL.pdf. Accessed on: Apr 14, 2009.

7. Working Group on Antiretroviral Therapy and Medical Management of HIV-Infected Children. Guidelines for the use of antiretroviral agents in pediatric HIV infection. 2009 Feb 23; p. 1-139. Available at: http:// aidsinfo.nih.gov/ContentFiles/PediatricGuidelines.pdf. Accessed on Apr 20, 2009.

8. Johnson VA, Brun-Vézinet F, Clotet B. Update of the drug resistance mutations in HIV-1: Dec 2008. Top HIV Med. 2008;16(5):138-145.

9. Schmidt B, Walter H, Moschik B, et al. Simple algorithm derived from a geno-/phenotypic database to predict HIV-1 protease inhibitor resistance. AIDS. 2000;14(12):1731-1738. 
10. Meynard JL, Vray M, Morand-Joubert L, et al. Phenotypic or genotypic resistance testing for choosing antiretroviral therapy after treatment failure: A randomized trial. AIDS. 2002;16(5):727-736.

11. Vray M, Meynard JL, Dalban C, et al. Predictors of the virological response to a change in the antiretroviral treatment regimen in HIV-1infected patients enrolled in a randomized trial comparing genotyping, phenotyping and standard of care (Narval trial, ANRS 088). Antivir Ther. 2003;8(5):427-434.

12. Hales G, Birch C, Crowe $\mathrm{S}$, et al. A randomised trial comparing genotypic and virtual phenotypic interpretation of HIVdrug resistance: The CREST Study. PLoS Clin Trials. 2006;1(3):1-9.

13. Romanelli F, Pomeroy C. Human immunodeficiency virus drug resistance testing: State of the art in genotypic and phenotypic testing of antiretrovirals. Pharmacotherapy. 2000;20(2):151-157.

14. Reeves JD, Piefer AJ. Emerging drug targets for antiretroviral therapy. Drugs. 2005;65(13):1747-1766.

15. Doms RW, Peiper SC. Unwelcomed guests with master keys: How HIV uses chemokine receptors for cellular entry. Virology. 1997;235(2): 179-190.

16. Gulick RM, Lelezari J, Goodrich J, et al. Maraviroc for previously treated patients with R5 HIV-1 infection. N Engl J Med. 2008;359(14): 1429-441.

17. Wood A, Armour D. The discovery of the CCR5 receptor antagonist, UK-427,857, a new agent for the treatment of HIV infection and AIDS. Prog Med Chem. 2005;43:239-271.

18. Fda.gov [homepage on Internet]. Silver Spring, MD: U.S. Food and Drug Administration. Available from: http://www.fda.gov/. Accessed on Jun 16, 2010.

19. Emea.europa.eu [homepage on the Internet]. London: European Medicines Agency; c1995 [updated 2009 Apr 30]. Available from: http://www.emea.europa.eu/. Accessed on May 6, 2009.

20. Pfizer. An open label pharmacokinetic, safety and efficacy study of maraviroc in combination with background therapy for the treatment of HIV-1 infected, CCR5. In: ClinicalTrials.gov [Internet]. Bethesda, MD: National Library of Medicine (US); c2000 [updated 2009 May 5]. Available from: http://clinicaltrials.gov/ct2/show/ NCT00791700: NCT00791700. Accessed on May 8, 2009.

21. Young W. Monogram Biosciences introduces new co-receptor tropism assay, profile, availability. August 2007. Available from: http://www.natap.org/2007/HIV/082107_04.htm. Accessed on Jun 21, 2009.

22. Wild CT, Shugars DC, Greenwell TK, McDanal CB, Matthews TJ. Peptides corresponding to a predictive $\alpha$-helical domain of human immunodeficiency virus type 1 gp41 are potent inhibitors of virus infection. Proc Natl Acad Sci U S A. 1994;91(21):9770-9774.

23. Lalezari JP, Henry K, O’Hearn M, et al. Enfuvirtide, an HIV-1 fusion inhibitor for drug-resistant HIV infection in North and South America. N Engl J Med. 2003;348(22):2175-2185.

24. Lazzarin A, Clotet B, Cooper D, et al. Efficacy of enfuvirtide in patients infected with drug-resistant HIV-1 in Europe and Australia. N Engl J Med. 2003;348(22):2186-2195.

25. Nelson M, Arastéh K, Clotet B, et al. Durable efficacy of enfuvirtide over 48 weeks in heavily treatment-experienced HIV-1 infected patients in the T-20 versus optimized background regimen only 1 and 2 clinical trials. J Acquir Immune Defic Syndr. 2005;40(4):404-412.

26. Xu L, Pozniak A, Wildfire A, et al. Emergence and evolution of enfuvirtide resistance following long-term therapy involves heptad repeat 2 mutations within gp41. Antimicrob Agents Chemother. 2005;49(3): 1113-1119.

27. Su C, Melby T, DeMasi R, Ravindran P, Heilek-Snyder G. Genotypic changes in human immunodeficiency virus type 1 envelope glycoproteins on treatment with the fusion inhibitor enfuvirtide and their influence on changes in drug susceptibility in vitro. J Clin Virol. 2006; 36(4):249-257.

28. Trottier B, Walmsley S, Reynes J, et al. Safety of enfuvirtide in combination with an optimized background of antiretrovirals in treatmentexperienced HIV-1infected adults over 48 weeks. J Acquir Immune Defic Syndr. 2005;40(4):413-421.
29. Mitsuya H, Weinhold KJ, Furman PA, et al. 3'-Azido-3'deoxythymidine (BW A509U): An antiviral agent that inhibits the infectivity and cytopathic effect of human T-lymphotropic virus type III/lymphadenopathy-associated virus in vitro. Proc Natl Acad Sci US A. 1985;82(20):7096-7100.

30. Tramontano E, Piras G, Mellors JW, Putzolu M, Bazmi HZ, La Colla P. Biochemical characterization of HIV-1 reverse transcriptases encoding mutations at amino acid residues 161 and 208 involved in resistance to phosphonoformate. Biochem Pharmacol. 1998;56(12): 1583-1589.

31. Rahim S, Fredrick LM, da Silva BA, Bernstein B, King MS. Geographic and temporal trends of transmitted HIV-1 drug resistance among antiretroviral-naïve subjects screening for two clinical trials in North America and Western Europe. HIV Clin Trials. 2009;10(2): 94-103.

32. Merluzzi VJ, Hargrave KD, Labadia M, et al. Inhibition of replication by a non-nucleoside reverse transcriptase inhibitor. Science. 1990; 250(4986):1411-1413.

33. Ren J, Stammers DK. Structural basis for drug resistance mechanisms for non-nucleoside inhibitors of HIV reverse transcriptase. Virus Res. 2008;134:157-170.

34. Nadler JP, Berger DS, Blick G, et al. Efficacy and safety of etravirine (TMC125) in patients with highly resistant HIV-1: Primary 24-week analysis. AIDS. 2007;21(6):1-10.

35. Madruga JV, Cahn P, Grinsztejn B, et al. Efficacy and safety of TMC125 (etravirine) in treatment-experienced HIV-1-infected patients in DUET-1: 24-week results from a randomized, double-blind, placebocontrolled trial. Lancet. 2007;370(9581):29-38.

36. Cohen CJ, Berger DS, Blick G, et al. Efficacy and safety of etravirine (TMC125) in treatment-experienced HIV-1-infected patients: 48-week results of a phase IIb trial. AIDS. 2009;23(3):423-431.

37. Lazzarin A, Campbell T, Clotet B, et al. Efficacy and safety of TMC125 (etravirine) in treatment-experienced HIV-1-infected patients in DUET-2: 24-week results from a randomized, double-blind, placebocontrolled trial. Lancet. 2007;370(9581):39-48.

38. Lataillade M, Kozal MJ. The hunt for HIV-1 integrase inhibitors. AIDS Patient Care STDS. 2006;20(7):489-501.

39. Jegede O, Babu J, di Santo R, et al. HIV type 1 integrase inhibitors: From basic research to clinical implications. AIDS Rev. 2008;10(3) $172-189$.

40. Steigbigel RT, Cooper DA, Kumar PN, et al. Raltegravir with optimized background therapy for resistant HIV-1 infection. N Engl J Med. 2008; 359(4):339-354.

41. Cooper DA, Steigbigel RT, Gatell JM, et al. Subgroup and resistance analyses of raltegravir for resistant HIV-1 infection. $N \mathrm{Engl} \mathrm{J} \mathrm{Med.}$ 2008;359(4):355-365.

42. Siskind R. Safety and effectiveness of raltegravir (MK-0518) in treatment-experienced, HIV-infected children and adolescents. In: ClinicalTrials.gov [Internet]. Bethesda, MD: National Library of Medicine (US); c2000 [updated March 9, 2009]. Available from: http://clinicaltrials.gov/ct2/show/NCT00485264. Accessed on May 6, 2009.

43. Roberts NA, Martin JA, Kinchington D, et al. Rational design of peptide-based HIV proteinase inhibitors. Science. 1990;248(4953):358-361.

44. Peterson ML, Wang Y, van der Laan MJ, et al. Virologic efficacy of boosted double versus boosted single protease inhibitor therapy. AIDS. 2007;21(12):1547-1554.

45. Gallant JE. Protease-inhibitor boosting in the treatment-experienced patients. AIDS Rev. 2004;6(4):226-233.

46. Sension M, Piliero PJ. Ritonavir-boosted protease inhibitors: Impact of ritonavir on toxicities in treatment-experienced patients. J Assoc Nurses AIDS Care. 2007;18(1):36-47.

47. Chen Z, Li Y, Schock HB, Hall D, Chen E, Kuo LC. Three-dimensional structure of a mutant HIV-1 protease displaying cross-resistance to all protease inhibitors in clinical trials. J Biol Chem. 1995;270(37): 21433-21436. 
48. Clotet B, Bellos N, Molina J, et al. Efficacy and safety of darunavirritonavir at week 48 in treatment-experienced patients with HIV-1 infection in POWER 1 and 2: A pooled subgroup analysis of data from two randomised trials. Lancet. 2007;369(9568):1169-1178.

49. de Meyer S, Vangeneugden T, van Baelen B, et al. Resistance profile of darunavir: Combined 24-week results from the POWER trials. AIDS Res Hum Retroviruses. 2008;24(3):379-388.

50. King NM, Prabu-Jeyabalan M, Nalivaika EA, Wigerinck P, de Béthune MP, Schiffer CA. Structural and thermodynamic basis for the binding of TMC114, a next-generation human immunodeficiency virus type 1 protease inhibitor. J Virol. 2004;78(21):12012-12021.

51. Tie Y, Boross PI, Wang YF, et al. High resolution crystal structures of HIV-1 protease with a potent non-peptide inhibitor (UIC-94017) active against multi-drug-resistant clinical strains. J Mol Biol. 2004;338(2): 341-352.

52. Prestiza ${ }^{\circledR}$ healthcare professionals full prescribing information. Mar 2009. Available from: http://www.prezista.com/prezista/hcp/full_ prescribing_information.html. Accessed on Jul 31, 2009.

53. Madruga JV, Berger D, McMurchie M, et al. Efficacy and safety of darunavir-ritonavir compared with that of lopinavir-ritonavir at 48 weeks in treatment-experienced, HIV-infected patients in TITAN: A randomised controlled phase III trial. Lancet. 2007;370(9581): $49-58$.

54. Turner SR, Strobach JW, Tommasi RA, et al. Tipranavir (PNU-140690): A potent, orally bioavailable nonpeptidic HIV protease inhibitor of the 5,6-dihydro-4-hydroxy-2-pyrone sulfonamide class. $J$ Med Chem. 1998;41(18):3467-3476.

55. Koh Y, Das D, Amano M, et al. Non-peptidyl small molecule protease dimerization inhibitors (PDIs): Molecular and structural ananlysis of their HIV-1 inhibition and interactions with protease monomer subunit. 15th Conference on Retroviruses and Opportunistic Infections (CROI 2008). 2008 Feb 3-6; Boston, MA.

56. Poppe SM, Slade DE, Chong KT, et al. Antiviral activity of the dihydropyrone PNU-140690, a new nonpeptidic human immunodeficiency virus protease inhibitor. Antimicrob Agents Chemother. 1997; 41(5):1058-1063.

57. Mazza F, Tronconi E, Valero A, et al. The non-peptidic HIV protease inhibitor tipranavir and two synthetic peptidomimetics (TS98 and TS102) modulate Pneumocystis carinii growth and proteasome activity of HEL299 cell line. J Eukaryot Microbiol. 2006;53 Suppl 1: S144-S146.

58. Squires K, McCallister S, Lazzarin A, et al. Tipranavir/ritonavir (TPV/r) demonstrates a robust resistance profile in multiple protease inhibitorexperienced patients: Correlation of baseline genotype and antiviral activity in BI 1182.52. The 2nd IAS conference on HIV pathogenesis and treatment (IAS 2003). 2003 Jul 13-16; Paris, France.

59. MacArthur RD, Richmond D, Crane LR. Tipranavir/ritonavir: Derivation of doses that achieve effective plasma concentrations above the $\mathrm{IC}_{50}$ for multiple PI-resistantHIV-1 viruses. 8th International Congress on Drug Therapy in HIV Infection (HIV 2006). 2006 Nov 12-16; Glasgow, Scotland.

60. Parkin N, Chappey C. Protease mutations associated with higher or lower than expected tipranavir (TPV) susceptibility based on the TPV mutation score. 13th Conference on Retroviruses and Opportunistic Infections (CROI 2006). 2006 Feb 5; Denver, CO.

61. Yeni P, MacGregor T, Gathe J, et al. Correlation of viral load reduction and plasma levels in multiple protease inhibitor experienced patients taking tipranavir/ritonavir in a Phase IIB trial: BI 1182.52. 10th Conference on Retroviruses and Opportunistic Infections (CROI 2003). 2003 Feb 10-14; Boston, MA.

62. Morello J, García-Gascó P, Rodríguez-Novoa S. Usefulness of the genotypic inhibitory quotient in HIV patients treated with tipranavir. The 4th IAS conference on HIV pathogenesis and treatment (IAS 2007). 2007 Jul 22-25; Sydney, Australia.

63. Bonora S, de Requena DG, Calcagno A, et al. Tipranavir (TPV) genotypic inhibitory quotient ( $\mathrm{gIQ}$ ) predicts early virological response to TPV-based salvage regimens. 13th Conference on Retroviruses and Opportunistic Infections (CROI 2006). 2006 Feb 5-8; Denver, CO.
64. Morse GD, Catanzaro LM, Acosta EP. Clinical pharmacodynamics of HIV-1 protease inhibitors: Use of inhibitory quotients to optimize pharmacotherapy. Lancet. 2006;6(4):215-225.

65. Hicks CB, Cahn P, Cooper DA, et al. Durable efficacy of tipranavirritonavir in combination with an optimised background regimen of antiretroviral drugs for treatment-experienced HIV-1-infected patients at 48 weeks in the Randomized Evaluation of Strategic Intervention in multi-drug reSistant patients with Tipranavir (RESIST) studies: An analysis of combined data from two randomised open-label trials. Lancet. 2006;368(9534):466-475.

66. de Requena DG, Bonora S, Calcagno A, et al. Tipranavir (TPV) genotypic inhibitory quotient predicts virological response at 48 weeks to TPV-based salvage regimens. Antimicrob Agents Chemother. 2008;52(3):1066-1071.

67. Salazar JC, Cahn P, Negra MD, et al. Predictors of response to TPV/r in pediatric patients: Results of BI1182.14/PACTG1051. 14th Conference on Retroviruses and Opportunistic Infections (CROI 2007). $2006 \mathrm{Feb}$ 25-28; Los Angeles, CA.

68. Mayers DL, Kohlbrenner VM, Dohnanyil C, et al. The inhibitory quotient (IQ) of tipranavir/ritonavir (tpv/r) in triple class experienced $\mathrm{HIV}^{+}$ patients: Results from bi 1182.52 . The 2nd IAS conference on HIV pathogenesis and treatment (IAS 2003). 2003 Jul 13-16; Paris, France.

69. Valdez H, McCallister S, Kohlbrenner V, Mayers D. Tipranavir/ritonavir (TPV/r) $500 \mathrm{mg} / 200 \mathrm{mg}$ BID drives week 24 viral load (VL) below 400 copies $/ \mathrm{mL}$ when combined with a second active drug (T-20) in protease inhibitor experienced $\mathrm{HIV}^{+}$patients. The 3rd IAS conference on HIV pathogenesis and treatment (IAS 2005). 2005 Jul 24-27; Rio de Janeiro, Brazil.

70. Naeger LK, Zheng JJ, Struble KA. Virologic response to tipranavir based on plasma concentration ( $\mathrm{Cmin}$ ) and baseline resistance parameters. 13th Conference on Retroviruses and Opportunistic Infections (CROI 2006) 2006 Feb 5-8; Denver, CO.

71. Flexner C, Bate G, Kirkpatrick P. Tipranavir. Nat Rev Drug Discov. 2005;4(12):955-956.

72. McCallister S, Valdez H, Curry K, et al. A 14-day dose-response study of the efficacy, safety, and pharmacokinetics of the nonpeptidic protease inhibitor tipranavir in treatment-naive HIV-1-infected patients. J Acquir Immune Defic Syndr. 2004;35(4):376-382.

73. Vourvahis M, Dumond J, Patterson K, et al. Effects of tipranavir/ ritonavir (TPV/r) on the activity of hepatic and intestinal cytochrome P450 3A4/5 and P-glycoprotein (P-gp): Implications for drug interactions. 14th Conference on Retroviruses and Opportunistic Infections (CROI 2007). 2007 Feb 25-28; Los Angeles, CA.

74. Baldwin JR, Boron MT, Wang J, Schneck DW, Hopkins NK. Effects of food and antacid on bioavailability of the protease inhibitor PNU140690 in healthy volunteers. 5th Conference on Retroviruses and Opportunistic Infections (CROI 1998). 1998 Feb 1-5;Chicago, IL.

75. Sabo JP, Huettner S, MacGregor TR, Wruck J. Evaluation of the bioavailability of tipranavir/ritonavir (TPV/r) $500 \mathrm{mg} / 200 \mathrm{mg}$ oral solution (OS): Comparison to TPV capsules and the influence of food intake in healthy volunteers. 6th International Workshop on Clinical Pharmacology of HIV Therapy (NATP 2005). 2005 Apr 28-30; Québec City, Canada.

76. Gathe J, Cooper DA, Farthing C, et al. Efficacy of the protease inhibitors tipranavir plus ritonavir in treatment-experienced patients: 24-week analysis from the RESIST-1 trial. Clin Infect Dis. 2006;43(10): 1337-1346.

77. Cahn P, Villacian J, Lazzarin A, et al. Ritonavir-boosted tipranavir demonstrates superior efficacy to ritonavir-boosted protease inhibitors in treatment-experienced HIV-infected patients: 24-week results of the RESIST-2 trial. 2006;43(10):1347-1356.

78. Salazar JC, Cahn P, Yogev R, et al. Efficacy, safety and tolerability of tipranavir coadministered with ritonavir in HIV-1 infected children and adolescents. AIDS. 2008;22(14):1789-1798.

79. Salazar JC, Cahn P, Giaquinto C, Yogev R, Mikl J. Efficacy and safety profile of APTIVUS oral solution or capsules co-administered with low dose ritonavir (APTIVUS/r) in treatment experienced children and teenagers after two years of treatment (Phase I/IIa study). XVII International AIDS Conference (AIDS 2008). 2008 Aug 3-8; Mexico City, Mexico. 
80. Division of AIDS table for grading the severity of adult and pediatric adverse events version 1.0. Dec 2004. Available from: http://www3. niaid.nih.gov/LabsAndResources/resources/DAIDSClinRsrch/PDF/ DAIDSAEGradingTable.pdf. Accessed on May 5, 2009.
81. Huang IC, Wu AW, Finnern HW, Thijs H, Gathe JC, Fairclough DL. Health-related quality of life and tolerability in treatment-experienced HIV-1-infected patients on tipranavir versus comparator regimens. Antivir Ther. 2008;13(1):15-25.

\section{Publish your work in this journal}

Therapeutics and Clinical Risk Management is an international, peerreviewed journal of clinical therapeutics and risk management, focusing on concise rapid reporting of clinical studies in all therapeutic areas, outcomes, safety, and programs for the effective, safe, and sustained use of medicines. This journal is indexed on PubMed Central, CAS,
EMBase, Scopus and the Elsevier Bibliographic databases. The manuscript management system is completely online and includes a very quick and fair peer-review system, which is all easy to use. Visit http://www.dovepress.com/testimonials.php to read real quotes from published authors.

Submit your manuscript here: http://www.dovepress.com/therapeutics-and-clinical-risk-management-journal 